\title{
eManaging Ambient Organizations in 3D
} Viktoria Skarler ${ }^{1}$

\author{
${ }^{1}$ Copenhagen Business School, CAICT, Center for Applied ICT, vs.caict@cbs.dk
}

Received 22 April 2008; received in revised form 28 April 2009; accepted 30 May 2009

\begin{abstract}
As we work in different constellations, levels, networks, and sub networks etc., we need to see networking in multiple dimensions, i.e. as ambient organizations. To summarize the problem statement the article is addressing whether or not there is an added value for interpreting the structure of collaboration by managing ambient organizations in 3D. By utilizing a cybernetic structure and combining a set of specific methods, a clear separation between narrative, content, and contexts of organizations can be determined and challenge the old traditional way of scientific and knowledge management. Take Stafford Beer's methodology of Team Syntegrity which is based on cybernetic system theory where he uses the model of regular polyedra's to manage knowledge creating and transferring by organizing individuals with a strict cybernetic structure, and see the group of individuals re-arrange themselves in a self-organizing way. This three dimensional approach of eManagement is used as a platform for suggestions of more 3D tools communication, knowledge-sharing and innovation through the use of the theory on Information Space by Max Boisot. This article focuses upon an ICT and organizational approach to show the difficulties in creating a network based on a virtual platform without equal contribution and interest by all parties involved and a common vision that motivate the individuals to be a part of the network. The support of this multi-dimensionality from information and communication technologies (ICT) moves us towards using the dynamics of social technologies as Web 2.0 (ff Web 3.0) and an intelligent semantic web. Both the ambient organization and the social technologies available raise the challenge of changing our mindset in order to be able to manage at all. Finally the paper applies these systems on the challenge of asymmetric information, globalization, and the economics at the bottom of the pyramid (BOP), which could be implemented or visualized as an e-business model.
\end{abstract}

Key words: Knowledge Management Systems, Cybernetic System Theory, ICT, 3-Dimensional Organizational Model, Business Innovation, Web 2.0, Semantic Web, Asymmetric Information, BOP-Market 


\section{Introduction}

Technology is changing at a fast pace; as are its validating sciences. It is a question of paradigm and change of paradigm. The system theorist Stafford Beer defines a paradigm as a model that exhibits a closed logic, which means that our attempts to break out of a fixed pattern of thinking are constantly defeated - by running headlong into our own premises. [1] Suggestions at present for contra paradigms on the other hand indicate that there are fundamental changes ahead regarding the creation and functioning of an organization and collaboration. Worth mentioning within the area of expert system is that a core fundamental logic in the form of meta-rules was rigidly designed to govern the evolving nature of the knowledge base. [6] To state the problem shortly the article is primarily addressing whether or not collaboration enabled through technological platforms could be simplified by adding an additional dimension; is there an added value by managing ambient organizations from three dimensions, hence collaborating in a heterarchical network structure? The article is proposing that we widen our horizon of interpreting how organizations and collaborations works today by simplify the structure of connections in a three dimensionality. The chosen methods illustrate the process of knowledge creation and collaboration. We will start by looking at some of these correlated changes of the information and communication technology (ICT) development and the network based organization. Continued by describing the two core methods seen as systems, could be integrated aiming at creating heterarchial (which gives a horizontal connectivity), ambient intelligent, cybernetically structured network groups. Finally the paper will illustrate the three dimensionality by Visualization I and II.

\section{Tendencies and Progression of New Technology}

Tendencies in different types of virtual collaborative environments and the ICT-sector as a whole, as for example the technology of Web 2.0 that changes our way of working, connecting, collaborating etc., urge us to switch our mindsets towards more participatory and heterarchical structure, while seeing the web as a technological platform. [20] Web 2.0 or soon even more utilized Web $3.0 \mathrm{ff}$. is the technology that enhances collaboration, technological enabler of more flexibility in working relationships. The importance of creating trust between partners (by making transparent and enlighten the possible threats of a technical platform where the members only meet virtual) is often a neglected priority. Without including an ethical discussion of defining the concept "trust", but trust that is built up between people for a long time, or trust among colleagues defined in a formal memorandum of understanding as rules agreed to in advance of working together. [24]

John Musser, Tim O'Reilly defines in "Web 2.0 Principles and Best Practices" that "Web 2.0 is a set of economic, social, and technology trends that collectively form the basis for the next generation of the Internet - a more mature, distinctive medium, characterized by user participation, openness, and network effects." [13] p. 12. They point to eight core patterns that need to be understood for grasping the idea and usability of this new way of functioning on the internet: Harnessing Collective Intelligence, Data Is the Next "Intel Inside", Innovation in Assembly, Rich User Experiences, Software Above the Level of a Single Device, Perpetual Beta, Leveraging the Long Tail, Lightweight Models and Cost-Effective Scalability. [13] pp.12-13.

Today our workspaces consist of managers and employees from different generations, where for instance a part of them work according to a structured hierarchical context. Others move towards the mindset of the Web 2.0 world which is based on participatory self-organizing heterarchies in an unstructured (wicked) context. There is a need for a more easily understandable, yet comprehensive, way of working and living in this "new" world. [19]

The new logic is related to today's communication system and language that produces, integrates and distributes different formats and modalities of words, images, sounds, etc. in our global culture. As Roccio Rueda explains, this new language is the hypertext (a counter-example to hypertext would be the emergence of word processing), producing radical changes on the writing and reading notions, promoting, consequently, new expressive and communicative models, which are related with a virtual or "electracy" era. She analyses the implications of the hypertext technology in our culture, like language, as a new writing technology through the use of Jacques Derrida's Deconstruction theory. She concludes that the relationship among technology and society is an interaction where the technology is society and society cannot be understood without its technical "fingerprints". [16]

\subsection{Working in Ambient Organizations}

The purpose of bringing the concept of ambient organizations into this setting is that it symbolically is suitable for explaining how the organizations of today function in multiple dimensions. This is a new turning point on the path where research and development efforts are exploring the socio-economical, political and cultural aspects of ambient intelligence. Distancing itself from previous features, like adaptive and anticipatory behavior. This latest change accentuates empowerment and participation of the user and tries to give back the control to the individual. [3]

Ambient organization is defined in a complementary way by e.g. Pouloudi, Bjorn-Andersen, Strasser, Wright et.al pointing in the same direction towards virtual organizations, their shape, form, structure and other qualifications, strengthened by technology. [17] They differentiate in their description of the term and model in some areas, 
whereby a pluralistic point of departure is suggested here, having all definitions in mind. In this article the term ambient organization therefore is used to describe the developmental change of organizational forms towards the network as the basic operating unit, enabled by integrated information systems that sustain business processes and boost flexibility, knowledge creation/sharing within and between organizational boundaries. [18] pp.100-111.

To remain competitive, businesses must evolve to become more agile and flexible. New types of Information Systems (IS) are designed to support the business process and value-oriented view of an organization in order to enable intra- and inter- organizational integration, as e.g.; Geographic Information Systems (Enterprise Geographic Information Servers), Expert Systems, whereas knowledge management systems are included in the field and also supported by Management Information Systems and Decision Support Systems. [12] Consequently organizational boundaries are becoming more fluid to include all units in a pervasive structure, that is to say working in multiple dimensions.

\section{ICT as an Enabler}

Traditionally we interpret our organizations to work according to the frequently used flat organization schemes (matrixes) that work in two dimensional hierarchies, when they should be visualized in multiple dimensions, as they function in today's reality of managing within an organization. Applying a multi-dimensional organization model (or system, seen from a system theoretical perspective) using ICT creates a possibility not seen before for a favorable visualization.

The idea behind Web 2.0 and semantic web is to realize this vision with the support of new technological evolutions, as for instance the architecture of a three dimensional organizational model (or system), often shown in the area of nano-technology. For example, the tagging system that flourishes on the web (del.icio.us, Digg, etc.) where people select common terms to describe information and data they find or post, which allows linking schemes and translation of their terms while gradually expanding the number of people, the sizes of the communities, the quality of the CWE (collaborative working environment), or other aspects of virtual forums. [4] As these webs understand one another automatically, the consequence is that the information, or data that flows between these connected nodes (working groups, individuals, etc.) becomes more symmetric as they are being identified and understood by automatic matching

\section{Methods for Shaping a 3D-Organization Model}

In order to enable the possibility of a multi-dimensional model of ambient organizations supported by ICT the ambition is to use two known theories that are based on a three dimensional (3D) thought, interpretation and visualization called System I (Team Syntegrity) and System II (Social Learning Cycle in Information Space), since I find those most important methods of use within a chain of systems, but thereby also indicating the need for more 3D systems (Inquiring Systems, Innovation Systems, Business Modeling Systems and Action Systems in resemblance to Stafford Beer's Viable Systems Model (VSM). The reason why this paper emphasizes on these two systems of initially five systems (further information regarding the total system chain: vs.caict@cbs.dk) in the VSM is that they very well illustrate the three dimensionality I am seeking.

When analyzing the structural functions suggested by Stafford Beers' Viable System Model (VSM), the observer can assess the system's viability in terms of the structural coupling of elements of the system with their changing niche. The VSM is composed of a set of operations (that develop the tasks related to their identity), a meta-system (that provides identity and cohesion to contained viable systems) and the environment it impacts and within which it sustains itself. Long-term societal survival happens as a result of effective interactions on all the levels of organization it finds itself coupled to in its interactions (i.e., the family, the eco-region, the nation, other countries, etc.) which account for not just present success but the welfare of future generations. [8] A social system is considered to be a learning organization in so far as it is proactive in managing its interactions with environmental complexity; it adapts to the challenges it faces and uses the opportunities offered by the environment to which it is coupled, by relating its internal dynamics to those perceived changes, benefiting rather than suffering from them. In a human community or society, this interaction inevitably involves a range of dimensions - local, regional and national governments. [14]

Sagi has presented a socio-cybernetic theory of "direct democracy" - where a society can govern itself successfully by monitoring and correcting its performance according to public mandates (he offers detailed criteria for implementation of the theory) [1]. It is the author's vision to interpret the concept of direct democracy within the subject of knowledge and information transfer and creation-process for the participants towards the direct contact nodes, without any layers, whereas the decision making process has a direct and democratic process.

\subsection{System I: Team Syntegrity}

System I is based on the principle of cybernetics which is founded across many different disciplinary areas and derives from mathematics, physiology, medicine, biology, anthropology, chemistry, philosophy, etc. Cybernetics is a discipline closely related to control theory and systems theory, known as the interdisciplinary study of the structure of 
regulatory systems. The view in this paper seeks the epistemology of cybernetics to discern a different (3D) pattern. Even one of the founders of cybernetics Warren McCulloch believed managerial cybernetics to be possible. Another contributor to cybernetics is Norbert Wiener defined the term "as the study of control and communication in the animal and the machine, whereas Stafford Beer states it "the science of effective organization", which is most applicable in this given context. [1]

The structure that we seek should reflect the notion of a perfect democracy, and it surely means that no individual, and initially no cause, should have ascendance over any other. Then in looking for polyhedra on which to construct democratic tensegrity models, we must consider only regular polyhedra: figures which have no top, no bottom, no sides - indeed no feature by which they may be specially oriented at all. These regular polyhedra may be distinguished by the arbitrary number of their faces: the tetrahedron (four), the cube (six), the octahedron (eight), the dodecahedron (twelve), and the icosahedron (twenty). The cube's faces are squares, and the dodecahedron's are pentagons; otherwise, the faces are all equilateral triangles [1]. According to Euler's law, there is a fixed relationship between the number of faces, the number of vertices that the faces define (that is, points where they join), and the number of edges that define the faces. Euler says that the number of faces plus the number of vertices is equal to the number of edges plus two. Then take a look at the models on offer: [1]

\begin{tabular}{|c|c|}
\hline Tetrahedron & $4+4=6+2=8$ \\
\hline Cube & $6+8=12+2=14$ \\
\hline Octahedron & $8+6=12+2=14$ \\
\hline Dodecahedron & $12+20=30+2=32:$ \\
\hline Icosahedron & $20+12=30+2=32:$ \\
\hline
\end{tabular}

Stafford Beer's Team Syntegrity [1] has its origin in cybernetic principles, exemplified in a polyhedral structure, preferable the icosahedron, where the 30 struts, the 20 faces and 12 vertices correspond to members, strategies, and topics respectively of a cooperation for organizing. [1] pp. 14-24. Thus it consists of 12 groups with five proponents and five critics in each in forming each topic, and of 20 groups of three proponents and three critics in forming strategies. It is designed as a large group of subgroups interacting in defining purpose, strategies and topics. It is open-ended; hence this system can be implemented in parallel, without interfering with existing organizational structure. An organization or network that adopts the architecture based on Team Syntegrity (and thereby cybernetic principles) will organize 12 teams corresponding to 12 topics, springing from one overall topic. Together with the 20 strategies it will form a coherent and sustainable three dimensional organization. [1] pp. 14-24. The finding of Stafford Beer is that each of these groups is self-organizing arbitrary numbers of topics and strategies, demonstrating the complexity of a 3D-organization. The self-organizing, created throughout the functionality behind the chosen polyhedral supports a democratic decision making process, are brought about by the interconnections and interdependencies that reverberated from the three dimensional structure.

The individuals involved in establishing each topic and strategy understand how the 12 topics evolve into the 20 strategies which inform and constrain the development of each strategy, and the same individuals have also directly participated in producing three other topics and three other strategies either as proponent or as critic. The reverberation set in motion during the initial syntegration processes continues into the goal-setting process, hence has sufficient redundancy and robustness to survive attrition and sufficient reverberation to accept and possibly inform new members (if that is the circumstance).

\subsubsection{Team Syntegrity Creates a Democratic Space}

Alex Bavelas (Bavelas, 1952) devised three measures for quantifying the organizational pattern. Take each member of a network, and count for minimal number of steps that person takes (according to the protocol established) to reach every other member. Some will be closer to one another compared to others, and one might need to ascend and descend various hierarchical levels, and that count for one member is their Minimal Connectivity. Having made the count for every person, the total is called Group Dispersion, and it is an unequivocal measure of the extent to which the group is dispersed. Each member now has a personal Relative Centrality (to calculate this, divide the Group Dispersion by the minimal connectivity of the individual). Third, the measure of Peripherality for members is the Relative Centrality of the most central member, minus the member's own. These three measures determine to what extent communication is democratic [1] pp. 13-14.

This contemplates the reason behind the choice of methods which is transmitted into systems (System I \& II) and correspond to the indication of working in multiple dimensions, namely in this occasion three dimensions, managing the asymmetric information flow. Asymmetric information can be explained as when the actors have different information [21].

What makes "a group" out of a random assortment of people? It surely has to do with motivation, and in some way also morale. That what brings people into cohesive groups is the shared information that had changed them into purposive individuals. Data themselves do not supply this cohesion: it is the interpretation of data that produces purpose, and it is the shared interpretation between individuals that produces group cohesion. In management and 
other areas there are several ways of scheduling team meetings to make sure the most effective use of time, simultaneously ensuring a continuation of reverberation (echo-effect of the knowledge creation loop) through the systems. [2] pp. 432-460. Joe Truss, who worked together with Stafford Beer in the U.K, has developed an illustrative figure that shows these interactions. [23] Below you see the evolution of the models:

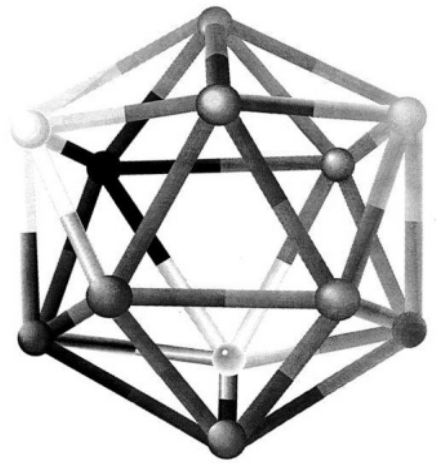

Fiqure 1: The Icosahedron, Stafford Beer

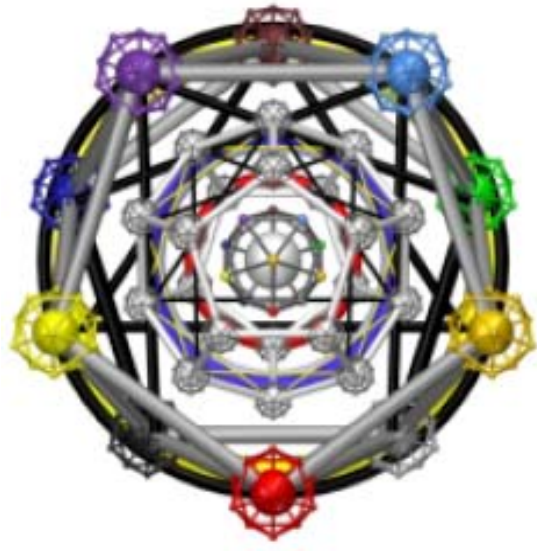

Figure 2: A reverberating system of a polyhedral

The 12 vertices and 20 faces are embedded in the dodecahedron. Inside the embedded dodecahedron is another icosahedron whose 20 faces correspond to the dodecahedron's 12 faces. Inside of this icosahedron lies another dodecahedron, and so on. The number of struts in each remains the same at 30, while the faces and vertices of the icosahedron and the dodecahedron change into each other. Seeing through any face of the outer icosahedron along a straight line to the centre, you are given a view of triangles contracting into points, thus expanding into smaller triangles lined up one inside the other, endlessly.

Visualizing all 20 faces at the same time going through this transformation exposes a perfectly symmetric diminution to regular polyhedral space within a regular polyhedral space, with each level summarizing the next through regular structural relationships and cooperation. Each level has a fractal self-similarity of shape and structure embedded and configured by the level before it afterward embedding and configuring the level after it, endlessly, granulated to smaller groups. The never ending recursions of equilateral triangles and pentagons, which are held in equilibration by an underlying regularity of shape maintained by the constant number of struts. [1] p. 14.

The methodology behind Team Syntegrity operates as follows, emphasizing on content with the support of a strong organized structure. When a member of a team takes on different roles in a given situation, as a participant, critic, and observer, the data (i.e. knowledge and information) crystallized during discussions will be transferred to other team discussions by diffusion of members with different perspectives as the participant takes upon the roles as critic and observer. I had the opportunity to facilitate a student workshop that went on in parallel with an IFIP conference in Maribor, Slovenia (2006), using a modification of the method of Stafford Beer's methodology Team Syntegrity, the participants were very suspicious of the model. But even if my small experience of handling a workshop based on Team Syntegrity, does not include understanding the Slovenian language and the amount of participants was insufficient according to the chosen Icosahedral model the method worked. The purpose was primarily to engage the students in the theme: Fair Globalization \& ICT and the second purpose aimed at introducing the participants to a different form of organizing the discussions towards a mutual goal, into groups. There is an underlying richness and power in the Icosahedral model which structure the working space through a democratic (equal participation, decision making \& delegation of the democratic process) collaboration. [2] pp. 239-269. In light of the discussion in this article it could be argued that the method face-to-face works extremely well and could create a basis for a platform structure, thus evolve electronically.

The outline above indicates the connection between the different constellations, creating a solid foundation, where the angles constitute a balance (S. Beer was inspired by Buckminster Fuller). This way of structuring a number of individuals, nodes (entities), or organizations increases the possibility of a more symmetric flow of data and thereby democratic creation of information and knowledge. We now turn to this process through the theory on Information Space by Max Boisot.

\subsection{System II: Social Learning Cycle in Information Space}

System II functions as tool for analyzing how the knowledge-flow as a Social Learning Cycle (SLC) in the Information Space (I-Space) within System I's syntegrations. According to Boisot organizations of today are data-structuring and data-sharing entities. In 1975 Williamson expressed that "[organizations] are made up of agents who successfully coordinate their actions by structuring and sharing information both with insiders - i.e., in hierarchies - and with outsiders - i.e., in markets" [5] pp. 169-170. Data structuring has two dimensions, according to Boisot, namely 
codification and abstraction. Codification is then illustrated as the creation of categories to which phenomena is allocated, together with rules of assignment [5] pp. 171-176.

Abstraction on the other hand can be explained as conceptualizing data or making the codified information into models. Codification and abstraction are cognitive strategies that any intelligent agent (Boisot uses the term agents, where e.g. Beer instead uses the concept members or team players) deploys in order to economize on dataprocessing costs. The two strategies strengthen one another and support each other in the process of making sense of the world by giving it a meaningful structure. They form two of the three dimensions of the information space (ISpace) as a conceptual framework. The sharing of data between one another is captured by a third dimension that describes data diffusion processes, where diffusion is explained as dissemination of the knowledge, and connects the economized with a target group [5] pp. 59-60.

Though we have entered a comparatively new form of society where data e.g. knowledge and information is the parameter that is accounted for in production next to capital and labor, mirroring the reality of society today. Boisot illustrates the new parameter in Information Space (I-Space) as data flourishing and identified by an evolutionary production function that explains the connectivity in reality, between capital, labor and data, thus adding one additional dimension compared to the neoclassical production function based on labor and capital only. But as there are transaction costs in making an economic exchange in labor and capital Ronald Coase (1937) could develop a theoretical framework for predicting when certain economic tasks are performed by firms and on the market, thus illustrates in his paper "The Nature of the Firm" from 1937. [7] These costs could either be internalized in the organization or externalized by sharing them with other organizations. Boisot tries to use his I-Space to show the different strategies in economizing on data through his Social Learning Curve (SLC).

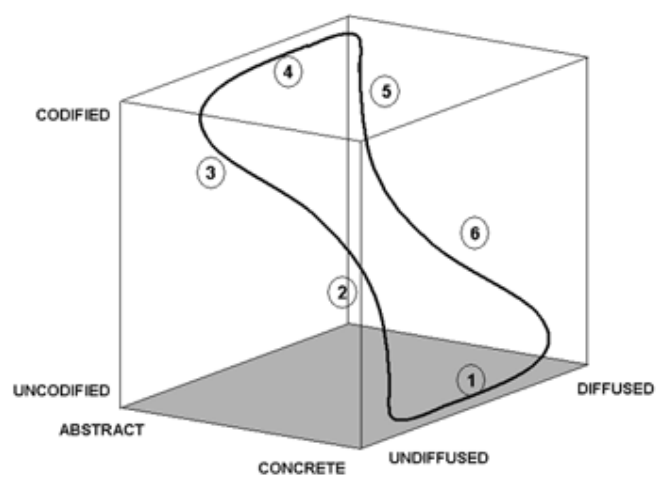

Figure 3: SLC in I-Space

Value, i.e. new knowledge and knowledge diffusion are created by movement in the I-Space through six phases by effectively activating all parts of the I-Space, indicated by the SLC. The six phases in SLC are as follows:

1. Scanning: Identify threats and opportunities, and gives patterns and insights.

2. Problem-solving: Creates structure out of the identified elements of data/information, and reduces uncertainty and challenges existing knowledge.

3. Abstraction: Generalization of obtained structure of data/information.

4. Diffusion: To share obtained knowledge with target groups.

5. Absorption: New knowledge becomes absorbed and made tacit, bodily, thus by learning-by-doing.

6. Impacting: Embedding knowledge in praxis, in products, techniques, and behavior.

In this model [5] knowledge is created, shared, used and demolished in three dimensions: codification, abstraction and diffusion. Boisot uses the metaphor of data being scattered (centripetal force) or gathered (centrifugal force) in a lector-magnetic field and then turned into information and knowledge according to social learning, culture, institution building, competencies and technology. The structuring and sharing of data are related. The more one can codify and abstract the data of experience, the more rapidly and extensively it can be transmitted to a given population of individuals. The work in teams (like in Team Syntegrity) is performed as a SLC in I-Space where the relationship is indicated by the curve of the in the I-Space. 
The point at the corner closest to you at the I-Space, a little to the left where the concrete knowledge and information gathering takes place on the curve, is a world in which knowledge is highly personal and hard to articulate, and is preferable transmitted by example rather than by explicit prescription thus shared on a face-to-face basis with trusted members over extended periods of time. The corner in the upper back, straight opposite you on the curve, by contrast, describes the world of e.g. bond traders, a world where all knowledge relevant to trading has been codified and abstracted into prices and quantities. Differences in the possibilities for structuring and sharing data can bring forth distinctive cultural practices and institutional arrangements. The features distinguishing such institutional arrangements from each other are e.g. to which degree: 1. relationships need interpersonal trust, 2. data is asymmetrically held, 3. specific types of exchange are recurrent and allows for emergent processes to function [5] pp. $70-75$

\subsection{The Connectivity of System I and II}

The purpose of illustrating the SLC in the I-Space is by screening the different perspectives, illuminate all possible gaps of gathering knowledge and information, thus cooperate and share knowledge between the connected topics. As teams in networks progresses through their life cycles, where both the problems they faces and solutions they explore becomes better structured and understood (by repeating the SLC in the I-Space), the more team processes becomes formalized. And as the knowledge the teams create needs to diffuse out to non-team members who do not share the team's tacit knowledge base, so team coordination gradually gives way to organizational processes. These processes will often diffuse the knowledge created by a team beyond the population that can be subjected to hierarchical bureaucratic control, so that such knowledge, by degrees, moves once more into the market region of the I-Space.

The skill required will consist of integrating the four different processes on networking, community, team, and organization, into a single seamless learning cycle. ICT itself is evolving in ways that will facilitate such integration. The distinction that is often made between old-style computing in which data is processed in a carefully preprogrammed and serial manner, and new-style computing, in which items of data is processed in a parallel and decentralized fashion, corresponds to two different locations in the I-Space (up to the left corner in I-Space, over to the right, down back in the middle, and finally to the right corner below). The first expresses a hierarchical approach to data processing that is institutionalized in human organizations as bureaucracies. The second takes data processing to be a horizontal activity requiring mutual adjustment.

\subsubsection{D in Organizational Models}

Using the I-Space as an analytical framework, one can explore the impact of ICT on organizational and cultural processes. ICT, by dramatically lowering the cost of data processing and transmission, will extend its influence well beyond organizations and culture. By production process imaginable, it is already accelerating and changing terms of trade-off between data and physical resources (labor and capital) Companies and businesses are organized and moves from the traditional hierarchical structure towards a more heterarchical shape. Some cultures are more suitable to profit from the internalization of trust relationships than others, as for example those that have a tradition of pluralism and tolerance [14].

By handling the structure in 3D the complexity explodes, but the teams are able to handle this proliferation of complexity through the self-organizing capabilities. This is in contrast to a hierarchical structure in 2D, which tries to handle complexity by reducing complexity. An organization from a $3 \mathrm{D}$ perspective illustrates that the entities is connected to multiple shapes of constellations in an ambient network. A 3D organization is a flexible organization that uses the self-organizing capabilities individuals belonging to different dimensions as a core competence emerges (and business advantage). 2D features and techniques will not have the same gravity as through the use of 3D models and 3D visualizations we are able to create concepts which can easily be learned by visual or tactile practicing. The benefits of utilizing an additional dimension are multi-faceted e.g.; increased design flexibility and design data integrity, increased accuracy of MRP (Material Requirements Planning) data, more effective identification and elimination of inefficiencies, concurrent engineering (CE), increased workload capacity and improved overall communication. The complexity is managed through system I, which creates a simple structure sketched in 3D that promotes a more participative, democratic space and stimulates and fosters self-organization in a heterarchical (un-structured dilemmas) order. System II handles the systematization of the unstructured dilemmas (context) that focus upon mapping the content. In other words, the visualization of sketching an organization in 3D illustrates how we actually work and clarifies the movement between the different dimensions; hence demolish the obstacles of the traditional structure.

The 3D-system is usable for a wide spectra of applications, thus it is crucial that the individuals in a team or alone, have the vision of seeing that organizations of today function in multiple dimensions. Combining Team Syntegrity and the SLC in I-Space we get a picture of a modern ambient organization in 3D (fig. 4):

The figure is illustrating my understanding of how the connection between System I \& II as they are functioning and working together in three dimensions. By combining System I \& II there is created a requisite variety for selforganizing thereby demolishing the complexity reduction paradigm of the traditional 2D organizational structure. The 
ensemble between the two 3D-models occurs in multiple and diverse dimensions, thus individual or groups are interplaying in different phases of the process of gathering and transferring knowledge or information, whereas the model is illustrating my understanding of how the connection between System I \& II as they are functioning and working together in three dimensions. By combining System I \& II there is created a requisite variety for selforganizing thereby demolishing the complexity reduction paradigm of the traditional 2D organizational structure. The ensemble between the two 3D-models occurs in multiple and diverse dimensions, thus individual or groups are interplaying in different phases of the process of gathering and transferring knowledge or information. The model at the top of the illustration is shaped as a molecule works up symmetries based on the icosahedron, whereas the model at the bottom illustrates the processing of knowledge and information in the three dimensional I-Space.
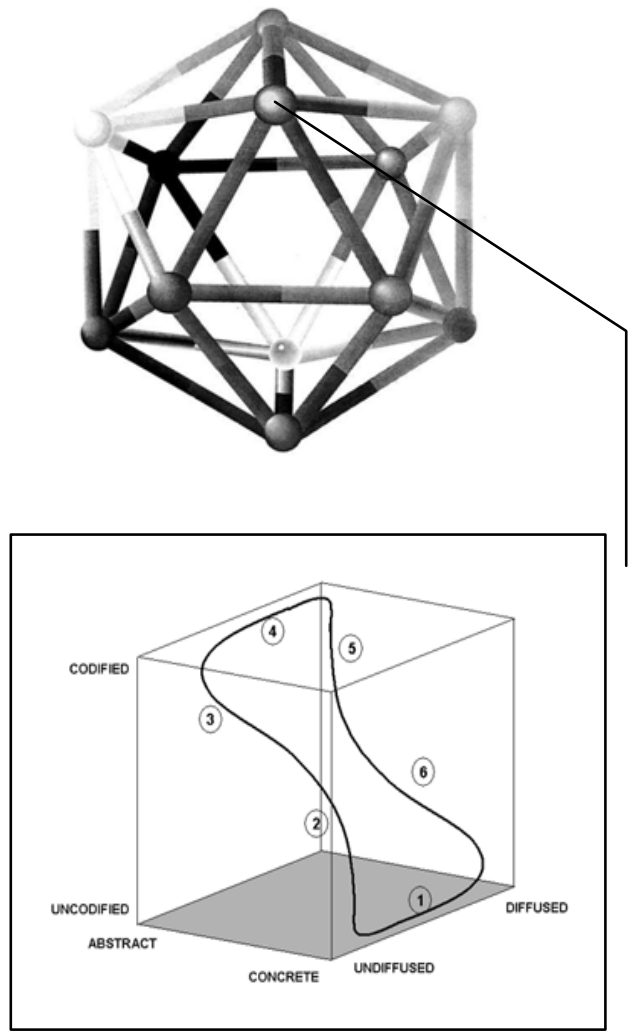

Figure 4: Illustration of a synthesis of models from; Boisot \& Beer

\subsection{Visualization I: BOP Markets and Knowledge Management}

The market at the Bottom of the Pyramid (BOP) as explained by Prahalad (2005) is a viable and valuable growth market; hence it could lead to poverty reduction, especially if all actors in the society are included in the cooperation (non-gorvenmental organizations [NGOs], MDCs, local people and organizations/businesses). The development of markets and effective business models at the BOP can support and encourage entrepreneurship in more than a financial way, thus a possibility for people to grow in many ways. [4] Then we place it on equal footing as a social transformation. For example, if we see development as following social transformation: "For the BOP consumers, gaining access to modern technology and good products designed with their needs in mind enables them to take a huge step in improving their quality of life." [15] p. 26

The first generation in the management of knowledge focused on the "right" way of structuring the flow of information to decision makers and the computerization of most business application striving to a technology enabled revolution dominated by the comprehended efficiencies of process reengineering. But newer theories on Knowledge Management and ICT point towards ambient intelligent network organization as a solution. George Akerlof introduced in his essay "The Market for Lemons" from 1970 the first formal analysis of markets with the informational problem known as adverse selection, exemplified by the market for used cars; "a lemon" - a colloquialism for a defective old car [21]. The ambient intelligent network screens out the "lemons", or should I say overload of information occurs, hence detects the signals where there is a need to create new knowledge (add more knowledge) or acquires more information.

The asymmetric information that flourishes in the BOP sphere - local or global - could be managed through a virtual and collaborative environment, supported by the structure of cybernetic constellations - and hence the imperfect 
information becomes as "perfect" as it can be (or symmetric) within a chosen market. The purpose of these networks and collaborative environments is to decrease the gap between developed and developing economies by giving access to the same information flow.

This eclectic model, connecting System I \& II as an integrated whole, could indicate that the probabilities - the beliefs - are conditional when it comes to information, hence any sensible way of representing uncertainties shall have a similar dependence. Solving the problem simply by focusing on ICT does not demolish the overall paradigm of economy as based on game theory. One aim is to suggest that to organize the knowledge- and information-flow by cybernetic and systemic management to create a democratic space and order. The intention is to create a three dimensional collaborative space that is self-organizing.

To develop such a new paradigm, we have to break out from long established premises, and to ask what should be taken as assumptions and what should be derived from the analysis. For example: market clearing could not be taken as an assumption, neither could the premise that a firm sells a good at a particular price to all consumers. You cannot begin the analysis even by assuming that in competitive equilibrium there would be zero profits. And the theory of Asymmetric Information verifies the need of managing the self-organizing virtual environments information and knowledge flow by structure of cybernetic, thus creating a framework with an open space within.

\subsection{Visualization II: Asymmetric Information and Beyond for BOP-markets}

Analyzing Visualization I more in dept Stiglitz describes the concept and theory behind asymmetric and imperfect information in his Nobel Prize lecture in 2001 [21] the discussion comes to whether or not it is possible to, via a 3D organizational model, make the information flow more symmetric. His research on the economics of information shows that whenever information is imperfect, in particular then there are information asymmetries where some individuals know something that others do not (which is often). Two different types of asymmetric information can be discerned; 1. Moral hazard, which signify that information about actions taking place are hidden for one of the actors, after initiating a contract or agreement and 2. adverse selection, which involves the differences of information that the actors contains (have) before a contract or agreement is initiated. When the information asymmetry are substantial there is a great risk that the market does not functions effectively, thus it could then be motivating to correct for topical market failure. [25]

When asymmetries of information occurs this indicates that different people know different things: employees know more about their work-capacity than the organization does; the car-salesman knows more about the car than potential buyers (example of the market for used cars: "lemons"), the individual buying insurance knows more about his health, than the insurance company and so on. Essential of a decentralized market economy is that people know different things, thus previous literature has emphasized on simple situations of information asymmetry - as described in previous paragraphs, the problems of information imperfections lies deeper. As e.g. if the person does not know about his true health condition; the insurance company, with a simple examination could know more about the individual's health condition than he does himself - and consequently gain advantage of information. [21] p. 17

Information imperfections and asymmetries of information are pervasive in every aspect of life and society. Political processes inevitably entail asymmetries of information; our political leaders are supposed to know more about threats to defense, about our economic situation, etc. than ordinary citizens. There has been a delegation of responsibility for day-to-day decision making, just as there is within a company. In "Making Globalization Work", Stiglitz attempts to show how globalization, properly managed, as it was in the successful development of much of East Asia, can do a great deal to benefit both the developing and the developed countries of the world. Attitudes toward globalization, and the failures and inequities associated with the way it has been managed, provide a Rorschach test for both countries and their people, revealing their fundamental beliefs and attitudes, their perspectives on the role of government and the market, the importance they attach to social justice, and the weight they put on noneconomic values. The "free market" economists are more inclined to believe that markets, by themselves, without government intervention, are efficient and that the best way to help the poor is simply to let the economy grow - and, somehow, the benefits will find its way down to the poor. This is interesting, since such beliefs have persisted, even as economic research has undermined their intellectual foundations. [22] pp. 61-65

Stiglitz emphasizes the connections between economic and cultural attitudes (cultural diversity, the environment, social justice, universal access to health care, consumer protection, etc.) to underline how much it matters to whom we entrust key aspects of economic decision making. Information affects decision making in every context and not only inside companies but also the political economy of information, namely the role of information in political process, and what could be collective decision making. [21] p. 3. Globalization encompasses many things: the international flow of ideas and knowledge, the sharing of cultures, global civil society, and the global environmental movement. As indicated here in Visualization I \& II, the problem lies not with globalization itself but in the way globalization has been handled; e.g. of how resources has been divided, where economics has been driving globalization, especially through the lowering of communication and transportation costs, and in the way that politics has shaped it. [22]

There are, according to Stiglitz, four pillars of successful development strategy and they are: markets, government, individuals, and communities. [22] pp. 3-24. A community is a group of people working together, often with help from 
government and nongovernmental organizations. In many developing countries, highly important collective action is at the local level, so communities really matter (to a successful development strategy). Trade liberalization is among the most controversial aspects of globalization; many see the alleged drawbacks - lower wages, growing unemployment, and loss of national sovereignty - as outweighing factors of the purported benefits of greater efficiency and increased growth. In part, free trade has not worked because we have not tried it: trade agreements of the past have been neither free nor fair. They have been asymmetric, opening up markets in the developing countries to goods from the advanced industrial countries without full reciprocation. Effective trade barriers have been kept in place, and this asymmetric globalization has put developing countries at a disadvantage. But even if trade barriers are brought down symmetrically, not everyone is equally positioned to take advantage of the new opportunities. For example, there is often a lack of infrastructure to bring their goods to the market, and it may take years for the goods they produce to meet the standards demanded by the advanced industrial countries. Trade liberalization can, when done fairly and when accompanied by the right measures and the right policies, help development. [22] pp. 9-17

\section{Logics are Conditional for Culture}

The overcoming of asymmetric information through System I \& II (Team Syntegrity \& Social Learning Curve) gives reasons and meaning to BOP for changing the western dominant logics that is affecting the less developed countries (or improving countries, as the specific vocabulary term I would like to use). The dominant logic of the western culture is in many counts affecting different logics in the whole world, on a local, regional and global level. Is it possible that knowledge management and ICT could solve the dilemma of asymmetric information? Is it a wicked (unstructured) problem in the Horst Rittel sense? If so, we need models that can handle wicked problems. [8] pp. 5565.

Cultural logics - connected to business innovation and the challenge of using differences as a core competitive advantage in our global economy (utilizing the intersection \& cultural logics in innovations) - deconstruction of the language. [16] Cultural logics are dynamic, shared predispositions that inform behavior and thought, which cannot predict specific actions, but they do lend a notion of regularity and continuity to behavior though post hoc analysis. Change is an integral part of culture, but such change must be reconciled with pre-existing cognitive schedules in a manner that allows for an intersubjective sense of cultural continuity, even if dramatic externally induced modifications. Cultural actors create their own unique cognitive worlds out of already cultured ideas, and the nature of cultural creativity itself thus binds individuals together through points of common reference, reinforcing and shaping cultural communities that could be related to a particular geographic territory. Similar like those of spiders, webs of cultural meaning are sticky. By cultural production, generally speaking, humans weave webs from which they have difficulties to escape from (and sometimes never) and change. At the level of internalized culture, individual cognitive patterns uniquely developed from available cultural resources, previous choices set down a path that delimits the wide range of current opportunities. [10] pp. 7-8

Patterns of cultural logics (illustrated by metaphors, historical narratives, religious beliefs, social sanctions, observed behavior, etc.) are received by individuals through the processes of socialization and ongoing social interaction, and also redefined through these very processes. Cultural logics are realized through practice, thus change and expand through interactions with others, differently conceived fields of reference, therefore must our formulations of other people's symbol systems be actor-oriented. [10] pp. 13-14

\section{Heterarchies - Organizations of Today}

Coming back to the 3D models of System I \& II this paradigm shift of the dominant western logic can be expressed in one concept, namely heterarchy.

Gerard Fairtlough is the developer of the triarchy theory, which states three fundamental ways of getting things done in organizations. Generally all organizations use a mixture of these three ways, but the extent to which they use each one can differ widely. Heterachy, is in an organizational context combined under the umbrella concept; Triarchy, where "responsible autonomy" and hierarchy together with heterarchy constitute the concept. [9] pp. 21-24. For the present, hierarchy is usually considered essential for all organizations, which is understandable since the way of interpreting how today's organizations function is not in a three dimensional way, but only in two. In a hierarchical organization it is mostly the individual at the top who learns, whereas creativity and innovation are limited to that of the individual at the top, thus an organization without learning will only survive in very stable conditions. In Stafford Beer's book Beyond Dispute, Joe Truss writes in the chapter "About Face, A turn for better planning" also on the possibility of a three dimensional organizational structure which gives the requisite interconnections without uncontrollable complexity. This goes along with the indication that the dominant organizational structure of today is moving towards a more participative form. [1] p. 344

Some organizations are trying to move away from a structure of hierarchical bureaucracy, which remains the dominant organizational structure, but more and more they will continue to move towards more participative structures. By doing so, they need to solve the problem of proliferating complexity resulting from reduced 
centralisation. Today the dominant structure is the two-dimensional matrix type organizational structure, which is providing to be too complex for large organizations. A 3D-system offers the requisite interconnections without unmanageable complexity, whereas the syntegrity icosahedron is from my perspective, a perfect viable architecture for the design of a 3D-organizational model.

Originally the term heterarchy [11] pp.89-93 is coined by Warren S. McCulloch early in 1943, where he in the article "A Heterarchy of values determined by the topology of nervous nets" [11], argues that all reflexes are activities of feed-back mechanisms, with consequences for the nervous system functioning, including all purpositive activity. McCulloch continues by saying that in addition to reflexes there exists within the central nervous system reverberating circuits which are called endromes, and he states that, "Without heterodromic activity reflexes would occur independently of one another. One may even ignore the asymmetry of ad version and aversion, but it is essential to remember that the circuit, whether regenerative or degenerative, must be closed for its activity to be purpositive." [11] p. 4

Warren S. McCulloch, stated following: "Given three dromes, each of which goes over one synaptic connection which is singly insufficient to fire its subsequent neuron but which may be reinforced from one other drome, an organism which fails to respond appetitively to any one of three sensory queues singly may respond to two by what appears to be a preference for one; and three such specious choices ma exhibit the circularity of the value anomaly. The threshold of the afferent neurons is such as to require impulses from the terminations of two axons, and that the heterodromic actions are summative instead of inhibitory." [11] p. 4. Heterarchy could be explained as an organisational shape between a network and hierarchy, which gives horizontal connection that permits different nodes to cooperate and allowing differing levels of connectivity, whilst individually optimizing diverse success criteria's. The difference between heterarchy and hierarchy lies in the element of sharing common goals in comparison with giving more power in decision making to the individuals higher up in the hierarchical structure. In his book "Beyond Dispute" Stafford Beer bring up the same notion heterarchy in opposition to heuristic hierarchy, where he narrate that hierarchy signify "government by priests" [1] pp. 240-241, where the antithesis is heterarchy implying; "the rule of an alien", as hetero means other or different, and the systems of different levels of embedment are alien to one another. [1] p. 241

In a heterarchial structure, on the other hand, each element or nodes, within the network, shares the same horizontal position of power and authority, with an equal vote and permission to connect. The process in a heterarchial structure handles complex flows of information more effectively compared to a hierarchical design. The heterarchial Wiki project function as a good example, hence are in some ways more democratic. The brilliance in interpreting the concept of heterarchy is in the process in which it permits the legitimate valuation of multiple skills, different knowledge or working routines without one having the power over the other.

\section{Tentative Conclusions and Future Explorations}

We need to challenge our traditional ways of structuring and managing organizations by implementing more tools that have similar possibilities to participate and collaborate, as the Wiki solution and Web 2.0 (even more probably Web 3.0 solutions). By witching our attitude and mindset where not only a higher possibility of working more participatory and collective is implemented, but also more importantly; shaping a more flexible structure that permits these shifts and demolishes the traditional hierarchal patterns. Semantic wikis virtually fosters personal knowledge management that at the same time gives people freedom to act within a formal structure in a collective and collaborative environment.

A future idea could be to develop further the theory and practice of the strategy-setting process, which fits in a variety of contexts, thus to explore the implications of the organizational scheduling system that evolves from using Team Syntegrity for strategic planning applications. This view could include computer software (or CWE) for operating electronic syntegrations on both topics and strategies, hence for scheduling members, teams, and group activities and accountabilities, interplayed with the other method mentioned in System II.

One direct reflection that comes to surface and needs to be explored further is the balance between physical and virtual work and collaboration (in all aspects - private, business and public sphere). Collaboration between individuals and organizations and the structure of working together and share information could generate many advantages for all parties involved. I would like to bring some light into the discussion that concerns the dilemma of totally depending on virtual cooperation at the personal, business, or civil society level and the consequences of creating trust between individuals without meeting face-to-face and by recommendations. The borders are getting erased both within the societal level but also of how we actually work. Is it possible to transfer discussions and sharing ideas totally on a virtual basis? How about building trust and sharing knowledge collaborating with multiple partners? Is there a need for a combination of both electronic and physical meetings and forums? Or can technological platforms be interchangeable by conversations face-to-face? What about our cultural differences and logics - are they being considered and taken as a positive or negative parameter (related to innovation this is an advantage). My hypothesis is that a semantic wiki enables the idea of System I \& II, functioning virtually, even though we should be aware that collaboration, sharing knowledge, and sustainable and long-term relationships need trust that comes through personal meetings for building this trust. 
Wikis are identified to be collaborative hypertext environments, or more suitable in this circumstance a knowledge management tool, that gives the possibility to individuals, groups and organizations to collaborate in a more participative way, where the information and knowledge flourishing symmetrically, being diffused and absorbed in the system. Like a chameleon the entities (nodes) within and between organizations, shift between the dimensions and type of interaction between multiple teams or participative organizations, with an ambient network functioning in 3D. This demands a structure which allows a flexibility within, that enables the individuals to function and work in multiple dimensions in the future, diminishing the asymmetry towards the BOP markets.

\section{Acknowledgments}

I would like to gratefully acknowledge the inspiration that I received during the years from Mr. Leif Bloch Rasmussen.

\section{References}

[1] S. Beer, Beyond Dispute: The Invention of Team Syntegrity. Chichester: John Wiley \& Sons Ltd., 1994.

[2] S. Beer, Decisions and Control: The Meaning of Operational Research and Management Cybernetics. Chichester: John Wiley \& Sons Ltd., 2000.

[3] Y. Benkler and H. Nissenbaum, Commons-based peer production and virtue, The Journal of Political Philosophy, vol. 14, no. 4, pp. 394-419, 2006.

[4] Y. Benkler, The Wealth of Networks: How Social Production Transforms Markets and Freedom. Yale University Press, 2006.

[5] M. H. Boisot, Knowledge Assets: Securing Competitive Advantage in the Information Economy. New York: Oxford University Press Inc., 1999.

[6] C. W. Churchman, System Analys, Stockholm: Norstedts Akademiska Förlag, 1968.

[7] R. H. Coase, The Nature of the Firm, in The Firm, the Market, and the Law. University of Chicago Press, 1990.

[8] J. Conklin, Wicked Problems and Social Complexity, in Dialogue Mapping: Building Shared Understanding of Wicked Problems, Chichester: John Wiley \& Sons Ltd., 2005.

[9] G. Fairtlough, The Three Ways of Getting Things Done: Hierarchy, Heterarchy \& Responsible Autonomy in Organizations. Triarchy Press, 2005.

[10] E. E. Fischer, Cultural Logics \& Economies: Maya Identity in Thought \& Practice. Austin: University of Texas Press, 2001.

[11] W. S. McCulloch, A Heterarchy of values determined by the topology of nervous nets, Bulletin of Mathematical Biophysics, vol. 7, pp. 89-93, 1945.

[12] L. Murphy, B. Thomas, H. Swayne, R. Metcalfe and J. Jones. (2009, March). An exploration of the skill and competence issues of an e-Business directed graphic design industry. International Journal of e-Business Management. [Online]. vol. 1, no. 1, pp. 22-36, 2007. Available: http://search.informit.comau/documentSummar; $\mathrm{dn}=905804038522380 ;$ res $=$ IELHSS.

[13] J. Musser, T. O'Reilly and the O'Reilly Radar Team: Web 2.0 Principles and Best Practices, 2006.

[14] J. Nahapiet and S. Ghoshal. (1998, April). Social capital, intellectual capital, and the organizational advantage. The Academy of Management Review. [Online]. vol. 23, no. 2, pp. 242-266. Available: http://www.jstor.org/stable/259373.

[15] C. K. Prahalad, The Fortune at the Bottom of the Pyramid. Upper Saddle River, NJ: Wharton School Publishing, 2005.

[16] Rocío Rueda Ortiz, Deconstruction theory applied to the network society: Risks and Challenges of the Network Society, ISBN: 0792372530, vol. 1, pp. 80-92, Ed., 2004.

[17] S. Roundy, M. Strasser, and P. K. Wright, Powering Ambient Intelligent Networks. Springer Berlin Heidelberg, 2005.

[18] D. Snowden, Complex acts of knowing: Paradox and descriptive self-awareness, Journal of Knowledge Management, vol. 6, no. 2, 2002.

[19] N. Spivack. (2007, July) The technical connectivity for the future: "Web 3.0 - Next Step for Web? ". Nova Spivack's Weblog. [Online]. Available: http://www.novaspivack.com/science/web-3-0-next-step-for-web.

[20] S. Ye, H. Chen and X. Jin, An empirical study of what drives users to share knowledge in virtual communities, in Knowledge Science, Engineering and Management. pp. 563-575, 2006.

[21] J. E. Stiglitz. (2001, December) Information and the change in the paradigm in economics, Nobel Prize Lecture. [Online]. Available: http://nobelprize.org/nobel prizes/economics/laureates/2001/stiglitz-lecture.pdf.

[22] J. E. Stiglitz, Making Globalization Work, W.W. Norton \& Company, Inc., New York, 2006.

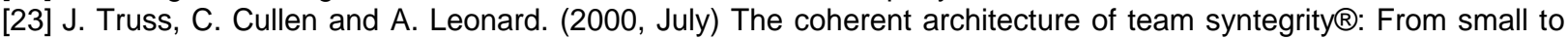
mega forms, World Congress of the Systems Sciences. [Online]. Available: http://www.syntegrity.com/web/ tseliveoffen.nsf/8dc07b89528557a2c1256aee0035c51f/c1256ab6001acd26c1256c7e0038e3e8/\$FILE/ISSS\%20 Artikel.pdf.

[24] W. Tsai and S. Ghoshal. (1998, August). Social capital and value creation: The role of intrafirm networks. The Academy of Management Journal. [Online]. vol. 41, no. 4, pp. 464-476. Available: http://www.jstor.org/stable/257085. 
[25] National Institute of Economic Research, Sweden. (2009, November) General Equilibrium Modeling - EMEC. [Online]. Available: http://www.konj.se/sidhuvud/inenglish/environmentaleconomics/cgemodeling.4.2479068e 10b3f7b60b980003617.html. 